\title{
Resultado clínico tardio da anuloplastia mitral sem suporte em crianças e adolescentes
}

\author{
Solange BORDIGNON*, Renato A. Karan KALIL*, João Ricardo M. SANT'ANNA*, \\ Paulo Roberto PRATES* ${ }^{\star}$ Edemar Manuel PEREIRA*, Ivo A. NESRALLA*
}

RBCCV $44205-318$

\footnotetext{
Bordignon S, Kalil R A K, Sant'Anna J R M, Prates P R, Pereira E M, Nesralla I A - Resultado clínico tardio da anuloplastia mitral sem suporte em crianças e adolescentes. Rev Bras Cir Cardiovasc 1996; 11 (4): $263-9$
}

RESUMO: O resultado clínico pós-operatório (p.o.) tardio da reconstrução da valva mitral sem suporte em crianças e adolescentes é analisado através da observação de uma série de 70 pacientes operados. Objetivou-se acompanhar a evolução clínica tardia após o tratamento de insuficiência mitral pelas técnicas reparadoras nos pacientes em fase de crescimento, avaliar a eficácia, a durabilidade e a reprodutibilidade do procedimento, bem como morbidade e mortalidade nas fases hospitalar e tardia. Consideraram-se, ainda, os resultados em relação à etiologia da doença e as causas de falha do método. De 1977 a 1995, foram operados 70 pacientes, sendo 36 casos do sexo feminino e 34 do masculino. A média de idade foi de $12,4 \pm 4,8$ anos ( $6 \mathrm{~m}$ a $18 \mathrm{a})$. Houve predomínio da etiologia reumática $(71,4 \%)$ sobre a congênita $(18,6 \%)$, a degeneraçāo mixomatosa $(8,6 \%)$ e a infecciosa $(1,4 \%)$. A classe funcional pré-operatória era ll em $32(45,7 \%)$ casos, III em $18(25,7 \%)$ casos e IV em $20(28,6 \%)$ casos. A técnica cirúrgica utilizada foi a anuloplastia simples tipo Wooler isolada em $58(82,9 \%)$ casos e associada a encurtamento de cordoalha em $12(17,1 \%)$. Em $21(30 \%)$ pacientes foram realizados procedimentos associados. O periodo de acompanhamento foi de $7 \mathrm{~m}$ a 17 anos, no p.o. A mortalidade hospitalar foi $4,3 \%$. Regurgitaçāo mitral foi descrita no intra-operatório em $21,4 \%$. Sopro sistólico de regurgitação mitral residual foi notado em $35(49,9 \%)$ pacientes, a maioria sem repercussão hemodinâmica. A classe funcional pós-operatória foi I em $73,9 \%$, II em $21,7 \%$ e III em $4,4 \%$. As curvas de análise atuarial mostraram aos 5 e 10 anos, respectivamente, probabilidade de sobrevida global de $89 \%$ e $79 \%$ e estimativa de permanecer livre de eventos cirúrgicos no periodo de $87 \%$ e $61 \%$ no grupo total, $88 \%$ e $56 \%$ no grupo de etiologia reumática e $91 \%$ no grupo de etiologia congênita para 5 e 10 anos. A insuficiência mitral pode ser tratada efetivamente por anuloplastia sem suporte anular protético, com resultados tardios comparáveis àqueles obtidos por técnicas mais complexas. Isto tem importância no tratamento de crianças e adultos jovens, especialmente no sexo feminino, quando se deseja evitar o implante de próteses mecânicas.

DESCRITORES: Insuficiência da valva mitral, cirurgia. Valva mitral, cirurgia. Cirurgia cardiaca, métodos. Valva mitral, cirurgia, criança. Valva mitral, cirurgia, adolescência.

Trabalho realizado no Instituto de Cardiologia do Rio Grande do Sul. Fundação Universitária de Cardiologia. Porto Alegre, RS, Brasil. Apresentado ao $23^{\circ}$ Congresso Nacional de Cirurgia Cardiaca. Recife, PE, 20 a 23 de março, 1996.

* Do Instituto de Cardiologia do Rio Grande do Sul.

Endereço para correspondência: Solange Bordignon. Instituto de Cardiologia do RS/FUC Unidade de Pesquisa. Av. Princesa Isabel, 395. Bairro Santana. CEP: 90620-001. Porto Alegre, RS, Brasil. Tel.[051] 223-5400. Fax.[051] 217-1358. 
Bordignon S, Kalil R A K, Sant'Anna J R M, Prates P R, Pereira E M, Nesralla I A - Resultado clínico tardio da anuloplastia mitral sem suporte em crianças e adolescentes. Rev Bras Cir Cardiovasc 1996; 11 (4):263-9.

\section{INTRODUÇÃO}

A doença reumática é a principal causa de doença cardiaca dos 5 aos 30 anos nos países subdesenvolvidos, correspondendo a $25 \%-40 \%$ de todas as doenças cardiovasculares e uma das maiores causas de morte em jovens. No Rio Grande do Sul, a prevalência estimada de cardiopatia reumática crônica é de 7:1000. Esta incidência é elevada quando comparada à média nos países subdesenvolvidos de 2:1000, enquanto que, em países desenvolvidos, é de 0,1:1000 (1).

A doença reumática é uma das diversas causas de insuficiência mitral, mas, sem dúvida, a mais freqüente. Em nossa experiência é aquela em que mais viável se torna a recuperação cirúrgica do aparelho valvar na população jovem, onde o implante das próteses artificiais é mais problemático, pois as biopróteses tendem a sofrer processos degenerativos precoces em pacientes de idade inferior a 35 anos (2) a as próteses metálicas exigem anticoagulação permanente. Diante destes fatos, o desenvolvimento de técnicas eficazes e duráveis de valvoplastia é de particular importância para o tratamento da doença valvar mitral.

A valvoplastia mitral constitui hoje o procedimento de preferência na correção da insuficiência mitral. Geralmente têm sido empregados anéis para o remodelamento da estrutura valvar (3-5). Na maioria das séries publicadas há predomínio de lesões não reumáticas (6-8). Poucos relatos referem a anuloplastia sem suporte (9-11).

A casuística aqui apresentada foi iniciada em 1977 com as técnicas reconstrutoras da valva mitral seguindo os princípios observados e propostos por Wooler acrescentando-se variações e modificações, objetivando avaliar a evolução clínica tardia após o tratamento da insuficiência mitral por técnicas reparadoras, sem o adjunto de suporte de anel ou barra, nos pacientes em fase de crescimento.

\section{CASUÍSTICA E MÉTODOS}

Entre 1977 e 1995, foram operados 70 pacientes, para a correção de insuficiência mitral isolada (sem estenose associada), pela técnica de Wooler simplesmente ou com modificações.

A análise dos dados foi completada em novembro de 1995, avaliando-se os pacientes, por períodos pós-operatórios, entre $7 \mathrm{~m}$ e 17 anos (média = $53,4 \pm 50,7 \mathrm{~m}) ; 6(8,6 \%)$ pacientes foram perdidos para acompanhamento após alta hospitalar e 3 $(4,3 \%)$ evoluíram para óbito ainda na fase hospitalar. Os demais $6(8,71 \%)$ tiveram sua evolução descrita no estudo. As idades variaram entre $6 \mathrm{~m}$ e 18 anos (média $=12,4 \pm 4,8$ ); predominou o sexo feminino com $36(51,4 \%)$ casos. A doença reumática esteve presente em $50(71,4 \%)$ casos. Em 13 $(18,3 \%)$ a etiologia era congênita, em $6(8,6 \%)$ casos degenerativa e em $1(1,4 \%)$ caso infecciosa (Tabela 1).

A classe funcional pré-operatória (NYHA) era IV em $20(28,6 \%)$ pacientes, III em $18(25,7 \%)$ e II em $32(45,7 \%)$.

Nenhum paciente foi operado em classe funcional I. As técnicas empregadas foram: anuloplastia tipo Wooler isoladamente em $58(82,9 \%)$ casos e associada a encurtamento de cordas tendíneas em $12(17,1 \%)$ casos. Havia 21 pacientes com procedimentos associados, como segue: $3(4,3 \%)$ valvoplastia aórtica, $9(12,9 \%)$ prótese aórtica, 4 (5,7\%)

TABELA1

VALVOPLASTIA MITRAL EM CRIANÇAS E ADOLESCENTES

\begin{tabular}{|c|c|c|c|}
\hline CASUÍSTICA & PERIOODO & $n$ & $\%$ \\
\hline & $1 / 77$ A $3 / 95$ & & \\
\hline \multirow[t]{2}{*}{ Idade } & $6 m$ a 18 anos & 70 & 100 \\
\hline & (média $=12,4 \pm 4,8 \mathrm{a})$ & & \\
\hline \multirow[t]{2}{*}{ Sexo } & Masculino & 34 & 48,6 \\
\hline & Feminino & 36 & 51,4 \\
\hline \multirow[t]{4}{*}{ Etiologia } & Reumática & 50 & 71,4 \\
\hline & Congênita & 13 & 18,6 \\
\hline & Degenerativa & 6 & 8,6 \\
\hline & Infecciosa & 1 & 1,4 \\
\hline \multirow[t]{4}{*}{ Seguimento } & $7 \mathrm{~m}$ a 15 anos & & \\
\hline & (média $=53,4 \pm 50,8 \mathrm{~m}$ ) & & \\
\hline & Pacientes & 64 & 91,4 \\
\hline & Pacientes Perdidos & 6 & 8,6 \\
\hline
\end{tabular}

$\mathrm{n}=$ número de pacientes; $\%$ = porcentagem; $\mathrm{m}=$ meses; $\mathrm{a}=$ anos 
Bordignon S, Kalil R A K, Sant'Anna J RM, Prates P R, Pereira E M, Nesralla I A - Resultado clínico tardio da anuloplastia mitral sem suporte em crianças e adolescentes. Rev Bras Cir Cardiovasc 1996; 11 (4):263-9 .

valvoplastia tricúspide, $4(5,7 \%)$ atrioseptoplastia e $1(1,4 \%)$ prótese aórtica e tricúspide (Tabela 2). Todos os pacientes foram submetidos ao procedimento que consistiu na redução da porção mural do anel valvar, obtida pela aplicação de pontos em $U$, de Poliester 2-0 ancorados em feltros de Teflon em cada comissura, de maneira a obter redução da circunferência do anel, sem comprometer a extensão da cúspide anterior. Este procedimento é similar ao descrito por KAY et al. (12), REED et al. (13) e WOOLER et al. (14).

Quando necessário, a correção do alongamento de corda tendínea foi realizada por sutura da corda comprometida na altura considerada adequada, baixando-a e fixando-a ao respectivo músculo papilar com fios de Poliester 2-0 ancorados em feltro de Teflon. O encurtamento da corda foi melhor realizado antes da anuloplastia.

Os pacientes foram operados por esternotomia mediana, sob circulação extracorpórea convencional, canulação das veias cavas e aorta e emprego de oxigenador de bolhas ou membrana descartáveis, com hipotermia moderada a $28^{\circ} \mathrm{C}-30^{\circ} \mathrm{C}$. Para proteção miocárdica foi empregada cardioplegia cristalóide, hipercalêmica e resfriamento da cavidade pericárdica com solução salina a $4^{\circ} \mathrm{C}$. As lesões cardiacas associadas eram corrigidas após a valvoplastia mitral.

O tempo médio de perfusão extracorpórea em toda a série foi $45,2 \pm 18,3$ e de $37,2 \pm 11,3$ nos casos sem lesões associadas.

Os tempos de isquemia miocárdica foram: 28,4 $\pm 14,3$ para toda a série e $21,8 \pm 7,1$ para a valvo-

TABELA 2

CLASSE FUNCIONAL

\begin{tabular}{lll}
\hline$n$ & $\%$ \\
\hline
\end{tabular}

Pré-operatória

$\begin{array}{ccc}\text { II } & 0 & 0 \\ \text { II } & 32 & 45,7 \\ \text { III } & 18 & 25,7 \\ \text { IV } & 20 & 28,6\end{array}$

Pós-operatória imediata

$\begin{array}{crc}\text { I } & 61 & 87,1 \\ \text { II } & 2 & 2,9 \\ \text { III } & 1 & 1,4 \\ \text { IV } & 0 & 0 \\ \text { Perdidos } & 6 & 8,6 \\ & & \\ \text { I } & 34 & 73,9 \\ \text { II } & 10 & 21,7 \\ \text { III } & 2 & 4,4 \\ \text { IV } & 0 & 0\end{array}$

$\mathrm{n}=$ número de pacientes; $\%=$ porcentagem plastia isoladamente. Testes de suficiência valvar foram realizados com solução salina gelada infundida na cavidade ventricular, através da valva mitral, enquanto na vigência da cardioplegia.

No pós-operatório, foram tomados os cuidados de rotina após a cirurgia cardiaca com circulação extracorpórea. Observou-se cuidadosamente a detecção precoce de eventuais sopros residuais de regurgitação mitral. A observação rigorosa de atividade reumática foi realizada e seu pronto atendimento estabelecido, devido às conseqüências desastrosas que pode ocasionar na valva operada.

Para análise clínica, os pacientes foram tabulados de acordo com idade, sexo, lesão cardíaca associada, procedimentos associados, classe funcional (NYHA), etiologia da lesão valvar, técnica de reconstrução valvar, grau de suficiência valvar ao teste transoperatório, presença e grau de sopro sistólico de regurgitação mitral residual no período pósoperatório, evolução clínica pós-operatória pela classe funcional, complicações tardias e reoperações. A ocorrência de óbitos nos primeiros 30 dias foi considerada mortalidade imediata. Os pacientes foram seguidos pessoalmente pelo cirurgião, ou no ambulatório do Hospital, ou por seu médico assistente e deste, os dados foram obtidos através de telefone ou carta. $\mathrm{Na}$ avaliação tardia foi considerado o grupo total de 46 pacientes e o grupo de reumáticos e congênitos, separadamente. Foi ainda analisado o subgrupo de etiologia reumática sem lesões associadas. Sobrevida sem eventos foi definida como probabilidade de o paciente estar vivo e livre de reoperação ou complicação maior relacionada à valva na época da avaliação. Pacientes reoperados por outras causas (disfunção de prótese aórtica) por não constituir falência do procedimento mitral não foram incluídos nas reoperações para fins estatísticos. Os resultados foram expressos como média \pm erro padrão. Probabilidade de sobrevida foi calculada pelo método atuarial.

\section{RESULTADOS}

Ocorreram $3(4,3 \%)$ óbitos imediatos, devidos a falência miocárdica, embolia pulmonar e falência de múltiplos órgãos. A mortalidade tardia foi $6(8,6 \%)$ casos e suas causas foram: falência de múltiplos órgãos (1 caso), endocardite bacteriana (1 caso) embolia pulmonar ( 2 casos), intoxicação digitálica (1 caso) e morte súbita (1 caso) (Tabela 3 ).

Treze pacientes foram reoperados a um tempo médio de seguimento de $56,5 \pm 46,2 \mathrm{~m}$.

Insuficiência cardiaca com insuficiência mitral importante esteve presente em 5 casos, endocardite bacteriana em 4 casos, cardite reumática em 2 
Bordignon S, Kalil R A K, Sant'Anna J R M, Prates PR, Pereira E M, Nesralla I A - Resultado clínico tardio da anuloplastia mitral sem suporte em crianças e adolescentes. Rev Bras Cir Cardiovasc 1996; 11 (4):263-9.

\begin{tabular}{|c|c|c|c|c|c|c|c|}
\hline MORTALIDA & $A D E$ & $n$ & $\%$ & TEMPO PO & IDADE & ETIOLOGIA & CAUSA \\
\hline Hospitalar & & 3 & 4,3 & & & & \\
\hline E.N.F. & (Reg 53388) & & & $11 \mathrm{~h}$ & $12 a$ & R & Emb.pulmonar \\
\hline T.S.C. & (Reg 67508) & & & $7 d$ & $6 m$ & C & FMO \\
\hline O.S.M. & (Reg 86147) & & & $4 h$ & $17 a$ & R & $\begin{array}{l}\text { Ch. cardiogênico } \\
\text { (Fal. miocárdica) }\end{array}$ \\
\hline Tardia & & 6 & 8,6 & & & . & \\
\hline M.A.D. & (Reg 217) & . & & $64 \mathrm{~m}$ & $22 a$ & A & FMO \\
\hline P.F.S.F. & (Reg 46428) & & & $121 \mathrm{~m}$ & $28 a$ & $\mathrm{R}$ & End. bacteriana \\
\hline C.C.B. & (Reg 65993) & & & $2 m$ & $16 a$ & $R$ & Emb.pulmonar \\
\hline L.E.S. & (Reg 67000) & & & $3 m$ & $12 a$ & R & Intox. digitálica \\
\hline H.A.S. & (Reg 6214) & & & $91 \mathrm{~m}$ & $24 a$ & R & Morte súbita \\
\hline O.C.S. & (Reg 33320$)$ & & & $83 m$ & $24 a$ & R & Emb.pulmonar \\
\hline
\end{tabular}

$\mathrm{n}=$ número de pacientes; $\%$ porcentagem; Reg. = registro; tempo po = tempo de pós-operatório; $\mathrm{h}=\mathrm{horas;} \mathrm{d}=$ dias; $\mathrm{m}=\mathrm{meses} ;$ $\mathrm{a}=$ anos; $\mathrm{R}=$ reumática; $\mathrm{C}=$ congênita; $\mathrm{Emb}=$ embolia; $\mathrm{FMO}=$ falência de múltiplos órgãos; $\mathrm{ch}=\mathrm{cheque} ; \mathrm{fal}=\mathrm{falência} ;$ end = endocardite; intox = intoxicação

casos, 1 caso de insuficiência cardiaca congestiva e embolia pulmonar e 1 caso de insuficiência aórtica.

$\mathrm{Na}$ avaliação tardia, de 46 sobreviventes sem reoperação, havia $34(73,9 \%)$ em classe funcional I, $10(21,7 \%)$ em classe funcional II e $2(4,4 \%)$ em classe funcional III. Nenhum paciente encontravase em classe funcional IV no final da pesquisa. No pré-operatório $54,3 \%$ dos pacientes encontravam- se em classe funcional III ou IV. Na última avaliação $95,6 \%$ dos pacientes acompanhados estavam em classe funcional I ou II. (Tabela 2).

A sobrevida atuarial foi $89 \%$ aos 5 anos e $79 \%$ aos 10 anos; incluindo a mortalidade imediata, a probabilidade de sobrevida pós-operatória sem eventos foi $87 \%$ aos 5 anos e $61 \%$ aos 10 anos (Gráfico 1).

\section{GRÁFICO 1}

PROBABILIDADE DE SOBREVIDA PÓS-OPERATÓRIA E POSSIBILIDADE DE SOBREVIDA SEM REOPERAÇÃO

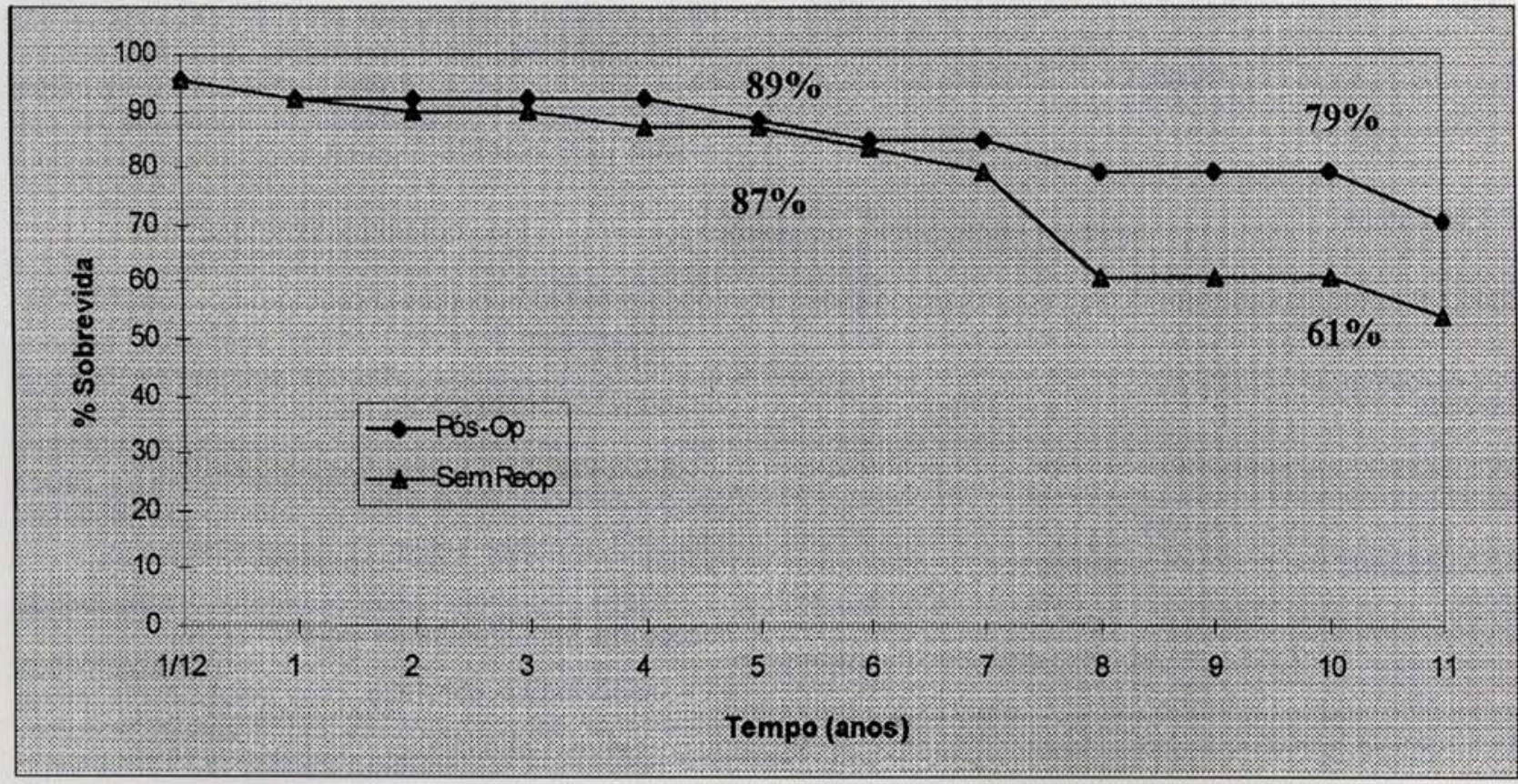


Bordignon S, Kalil R A K, Sant'Anna J R M, Prates P R, Pereira E M, Nesralla I A - Resultado clínico tardio da anuloplastia mitral sem suporte em crianças e adolescentes. Rev Bras Cir Cardiovasc 1996; 11 (4):263-9.

A durabilidade do procedimento em etiologia reumática, incluindo-se aquelas com lesões associadas, foi $89 \%$ aos 5 anos e $56 \%$ aos 10 anos. Quando avaliada a durabilidade do procedimento em etiologia reumática, sem lesōes associadas, a mesma foi, respectivamente, $88 \%$ e $58 \%$ aos 5 anos e 10 anos. A durabilidade do procedimento em etiologia congênita foi $91 \%$ aos 5 anos e 10 anos.

\section{COMENTÁRIOS}

Atualmente, a valvoplastia constitui o método de preferência para o tratamento da insuficiência mitral. Existe consenso geral quanto ao fato de que é preferivel conservar uma valva natural, embora algo imperfeita, a substitui-la por dispositivos artificiais (15). Muitas séries publicadas incluem, predominantemente, casos de etiologia degenerativa $(6,7,12,16)$. Quase sempre, anel ou barra posterior é implantado $(3,5,8)$. A utilização de anéis tem sido justificada sob o conceito de remodelamento do anel, julgado por alguns como sendo de importância na manutenção de um reparo adequado e durável $(8,17,18)$. Poucos relatos se referem a anuloplastia sem suporte $(11,19,20)$. Datados de 1938 , trabalhos experimentais e clínicos visavam obter um método efetivo de correção da insuficiência mitral, tanto por simples reparo valvar ou por implante de enxertos ou próteses $(21,22)$. As técnicas propostas para corrigir a regurgitação apresentam em comum o objetivo de reduzir o diâmetro do anel valvar, associando-se a este a correção das cordas rotas ou alongadas.

Desde o advento da circulação extracorpórea, a abordagem da valva mitral por visão direta proporcionou a avaliação da lesão, bem como o resultado do procedimento reconstrutivo. A primeira correção de insuficiência mitral realizada com sucesso, utilizando circulação extracorporea, foi realizada por LILLEHEI et al. (23)

KAY et al (12) empregaram técnicas reconstrutivas para insuficiência mitral secundária a dilatação do anel ou a rotura de cordas tendíneas, salientando a necessidade de não comprometer a cúspide septal nas suturas.

A anuloplastia tipo Wooler (WOOLER et al. ${ }^{(14)}$ ) é um método que visa ao encurtamento da cúspide mural, a qual será fixada junto às comissuras por suturas ancoradas em feltros de Teflon. A diferença da técnica de Kay reside apenas quanto ao tipo de sutura e o ancoramento da mesma para prevenir desgarramento posterior.

Contudo, após a publicação do estudo de STARR \& EDWARDS (24), em 1961, sobre a substituição valvar por prótese metálica de bola, tornou-se tec- nicamente mais fácil substituir a valva enferma do que tentar repará-la. Em conseqüência, a valvoplastia mitral foi relegada a planos secundários. Por outro lado, o conceito de que seria preferivel a manutenção da valva natural em lugar de próteses artificiais foi mantido e defendido por outros (10). À medida em que as observações tardias dos centros que persistiam no emprego de técnicas reconstrutivas passaram a ser divulgados, no final da década de 70 e ínicio da de 80 , houve uma retomada do emprego da anuloplastia simples e do implante de anéis rígidos ou semi-rígidos pré-moldados $(4,25)$. A complexidade anatômica e funcional da valva mitral tem sido citada como responsável pelo desencanto inicial de muitos cirurgiões com as técnicas reconstrutivas. ANTUNES et al (26) apresentaram claramente os progressos nos resultados, após o melhor entendimento e domínio dos métodos, mostrando a curva de aprendizado e afirmando que o reparo da valva mitral é possível na quase totalidade das crianças com insuficiência mitral reumática. Neste estudo, deu-se preferência para o implante de anéis rígidos em $69 \%$ dos casos, já que de 100 pacientes anteriormente submetidos à anuloplastia simples, apenas $\mathbf{4 0}$ mantiveram-se com função aceitável após 5 anos. Esta não tem sido a nossa experiência com a técnica de Wooler e acreditamos desnecessário o implante de anéis ou barras posteriores para obtenção de boa e durável suficiência valvar.

A reconstrução da valva mitral insuficiente por prolapso, através da plicatura das cúspides e da anuloplastia póstero-medial, tem sido considerada efetiva por alguns autores $(18,20)$, mas com um índice de recorrência de $16 \%$ nos primeiros anos (27). A casuística apresentada nesta série é de 70 pacientes operados entre 1977 a 1995. Foram incluídos neste estudo pacientes com idade de $6 \mathrm{~m}$ a 18 anos (média de 12,4 $\pm 4,8 \mathrm{a}$ ). Considerando-se que a opção por prótese em pacientes com idade menor do que 35 anos representa um sério dilema pela degeneração precoce das biopróteses ou pela necessidade de anticoagulação nas próteses mecânicas, a alternativa de recuperação do aparelho valvar é particularmente importante. Além disso, a incidência de $51,4 \%$ do sexo feminino, com probabilidade de gravidez no período pós-operatório tardio, torna mais clara a vantagem da manutenção da valva natural, pois a presença de prótese mecânica associada a anticoagulantes converte a gravidez em alto risco.

A etiologia reumática foi predominante nesta experiência $(71,4 \%)$, possívelmente devido a sua alta prevalência na nossa população. A maioria dos pacientes foi operada em classe funcional III ou IV $(54,3 \%)$. Dos 46 pacientes acompanhados tardiamente 44 ou $95,7 \%$ encontram-se em classe funcional I ou II, demonstrando a melhora obtida pelo 
Bordignon S, Kalil R A K, Sant'Anna J R M, Prates P R, Pereira E M, Nesralla I A - Resultado clínico tardio da anuloplastia mitral sem suporte em crianças e adolescentes. Rev Bras Cir Cardiovasc 1996; 11 (4):263-9.

método. Outra evidência objetiva da simplicidade do procedimento de anuloplastia pode ser notada observando-se os tempos de perfusäo e de isquemia miocárdica, respectivamente, $37,2 \pm 11,3$ min e 21,8 $\pm 7,1$ min consideravelmente baixo, em comparação com qualquer outra operação da valva mitral. Dentre os 13 pacientes que evoluíram para reoperação, $38,5 \%$ foram por disfunção mitral, $30,7 \%$ por endocardite bacteriana, $15,4 \%$ por novos casos de cardite reumática, $17,7 \%$ por disfunção de valva aórtica e $7,7 \%$ por insuficiência mitral e embolia pulmonar. Recomendamos a anuloplastia simples nos casos de insuficiência congênita ou reumática, devido à dilatação do anel, associada ou não a rotura ou alongamento de cordas; contudo, é fundamental a prevenção de novos surtos de atividade reumática ou de episódios de endocardite infecciosa, causas mais freqüentes de insucessos a longo prazo.

RBCCV $44205-318$

Bordignon S, Kalil R A K, Sant'Ana J R M, Prates P R, Pereira E M, Nesralla I A - Late outcome of unsupported annuloplasty as surgical treatment of mitral insufficiency in children and adolescents. Rev Bras Cir Cardiovasc 1996; 11 (4): 263-9.

ABSTRACT: Late post-operative clinical results for treatment of mitral regurgitation (MR) in patients under 18 years-old by annuloplasty without ring or posterior support are presented. From 1977 to 1995,70 patients: 36 female and 34 male, mean age $12.4 \pm 4.8$ y ( $6 \mathrm{~m}$ to $18 \mathrm{y})$, with pure MR were submitted to an Wooler type of annuloplasty. None received ring or annular support. Twelve $(17.1 \%)$ had chordal shortening associated. Ethiology was rheumatic $71.4 \%$, congenital $18.6 \%$, myxomatous $8.6 \%$ and infectious $1.4 \%$. Preoperative functional class was II: 32 cases $(45.7 \%)$, III: $18(25.7 \%)$, IV: $20(28.6 \%)$. Twenty-one patients $(30 \%)$ had associated procedures: on aortic valve $12(15.2 \%)$, tricuspid $4(5.7 \%)$, ASD $4(5.7 \%)$ and aortic and tricuspid $1(1.4 \%)$. Mean perfusion time was $45.2 \pm 18.3 \mathrm{~min}$ for the whole group and $37.2 \pm 11.3 \mathrm{~min}$ for annuloplasty alone. Mean ischemic time was $28.4 \pm 14.3 \mathrm{~min}$ and $21.8 \pm 7.1 \mathrm{~min}$ respectively. Follow-up time ranged from 7 months to 18 years. Mortality was $4.3 \%$ ( 3 cases) in the early postoperative and $8.6 \%$ ( 6 cases) in the late follow up. Early residual regurgitation was found in 15 patients $(21.4 \%)$ and $50.0 \%$ in 35 . Thirteen $(18.6 \%)$ were reoperated at a mean p.o. time of $56.2 \pm 46.2 \mathrm{~m}$. Causes for reoperation: primary MR $5(38.5 \%)$, endocarditis $4(30.7 \%)$, stenosis $2(15.4 \%)$, aortic valve disfunction $1(7.7 \%)$, pulmonary embolism $1(7.7 \%)$. Late evaluation in 46 non-operated survivors: 34 were in functional class I $(73.9 \%), 10$ in II $(21.7 \%)$ and 2 in III (4.3\%). Actuarial survival was $93 \pm 3 \%$ at 5 years and $80 \pm 7 \%$ at 10 years. Event-free survival was $89 \pm 4 \%$ and $61 \pm 10 \%$ at 5 and 10 years. For rheumatic ethiology, event-free survival was $80 \pm 8 \%$ and $55 \pm 16 \%$ and, for congenital MR, $90 \pm 9 \%$ at 5 and 10 years p.o. Simple, unsupported annuloplasty (Wooler type), alone or associated to chordal shortening is an effective and reproducible procedure for MR in children and adolescents. Morbidity and mortality are low in relation to other techniques and prosthetic replacement. Failures were mainly related to rheumatic carditis and infective endocarditis. In growing up patient under 18 years old, this technique would be recommended because it may allow unrestricted development of the mitral orifice.

DESCRIPTORS: Mitral valve insufficiency, surgery. Mitral valve, surgery. Heart surgery, methods. Mitral valve, surgery, child. Mitral valve, surgery, adolescence.

\section{REFERÊNCIAS BIBLIOGRÁFICAS}

1 Gus I, Zaslavsky C, Seguer J M, Machado R S - Febre reumática aguda. Rev Pesq Méd FFFCMPA 1993; 27: $35-43$

2 Magilligan D J, Lewis Jr J W, Lee M W, Alam M, Riddle J M, Stein P D - Spontaneous degeneration of porcine bioprosthetic valves. Ann Thorac Surg 1980; 30: 259-66.

3 Braile M D, Ardito R V, Pinto G $\mathrm{H}$ et al. - Plástica mitral. Rev Bras Cir Cardiovasc 1990; 5: 86-98.

4 Carpentier A, Chauvand S, Fabiani J N et al. -
Reconstructive surgery of mitral valve incompetence: ten year appraisal. J Thorac Cardiovasc Surg 1980; 79: $338-48$.

5 Cooley D A, Frazier O H, Norman J C - Mitral leaflet prolapse: surgical treatment using a posterior annular collar prosthesis. Cardiovasc Dis Bull Texas Heart Inst 1976; 3: 438-44.

6 Cosgrove D M, Chavez A M, Lytle B W - Results of mitral valve reconstrution. Circulation 1986; 74 (Suppl 1): 182-7.

7 David T E, Armstrong S, Sun Z, Daniel L- Late results of mitral valve repair for mitral regurgitation due to 
Bordignon S, Kalil R A K, Sant'Anna J R M, Prates P R, Pereira E M, Nesralla I A - Resultado clínico tardio da anuloplastia mitral sem suporte em crianças e adolescentes.

Rev Bras Cir Cardiovasc 1996; 11 (4):263-9.

degenerative disease. Ann Thorac Surg 1993; 56: $7-14$

8 Deloche A, Jebara V A, Relland J Y M et al. - Valve repair with Carpentier techniques. J Thorac Cardiovasc Surg 1990; 99: 990-1002.

9 Arruda M B, Moraes C R, Lagreca J R et al. - Anuloplastia mitral. Arq Bras Cardiol 1974; 27 (Supl 1): 32 (Resumo).

10 Kalil R A K, Lucchese F A, Prates P R et al - Late outcome of unsupported annuloplasty for rheumatic mitral regurgitation. JACC 1993; 22: 1915-20.

11 Pomerantzeff, P M A, Amato M, Stolf N A G et al. Experiência com plástica de valva mitral. Arq Bras Cardiol 1985; 45 (Supl 1): 156. (Resumo).

12 Kay J H, Zubiate P, Mendez M A, Vanstrom N, Yokohama $T$ - Mitral valve repair for significant mitral insufficiency. Am Heart $J$ 1978; 96: 253-62.

13 Reed G E, Tice D A, Clauss R H - Asymetric exaggerated mitral annuloplasty: repair of mitral insufficiency with hemodynamic predictability. J Thorac Cardiovasc Surg 1965; 49: 752-61.

14 Wooler G H, Nixon P G F, Grimshaw V A, Watson D A - Experience with the repair of the mitral valve in incompetence. Thorax 1962; 17: 49-57.

15 Kalil R, Lucchese F A, Prates P R - Anuloplastia sem suporte para tratamento da insuficiência mitral reumática. Rev Bras Cir Cardiovasc 1992; 7: 18693.

16 Cohn L M \& Collins Jr J J - Local hypothermia for myocardial protection. Ann Thorac Surg 1974; 17: 135.

17 Duran C G, Pomar J L, Revuelta J M et al. - Conservative operation for mitral insufficiency: critical analysis supported by postoperative hemodynamic studies of 72 patients. J Thorac Cardiovasc Surg 1980; 79: 326-37.
18 Lessada A, Carbone C, Romano M et al. - Mitral valve repair: results and the decision - making process in reconstruction. J Thorac Cardiovasc Surg 1990; 99: $622-30$

19 Kalil R A K, Prates P R, Lucchese F A et al - Valvoplastia para insuficiência mitral: técnicas e resultados. Arq Bras Cardiol 1981; 37: 315-9.

20 Paixão E L, Burgos F J C, Piegas L S et al. - Plastia mitral: resultados tardios. Arq Bras Cardiol 1986; 47 (Supl 1): 124-8.

21 Merendino K A, Thomas G I, Jesseph J E, Herron P W, Wintercheid L C, Vetto R R - The open correction of rheumatic mitral regurgitation and / or stenosis, with special reference to regurgitation treated by posteromedial annuloplasty utilizing a pump-oxygenator. Ann Surg 1959; 150: 5-22.

22 Nunley D L \& Starr A - The evolution of reparative techniques for the mitral valve. Ann Thorac Surg 1984; 37: 393-7.

23 Lillehei C W, Gott V L, Dewall R A, Varco R L - Surgical correction of pure mitral insufficiency by annuloplasty under direct vision. Lancet 1957; 77: 446-9.

24 Starr A \& Edwards M D - Mitral replacement: clinical experience with a ball-valve prosthesis. Ann Surg 1961; 154: 726-40.

25 Gregori F, Silva S S, Baba K - Um novo modelo de anel protético para pacientes com insuficiência valvar mitral: relato de 2 casos. Arq Bras Cardiol 1988; 50: 417-20.

26 Antunes M J, Colsen P R, Kinsley R H - Mitral valvuloplasty: a learning curve. Circulation 1983; 68 (Suppl 2): 70-5.

27 Penkoske P A, Ellis Jr F H, Alexander S, Watkins Jr E - Results of valve reconstruction for mitral regurgitation secondary to mitral valve prolapse. $A m$ J Cardiol 1985; 55: 735-8. 